\title{
APPLICATION OF A NETWORKED DECENTRALIZED MPC TO SYNGAS PROCESS IN OIL INDUSTRY
}

\author{
S. Longhi* R. Trillini ${ }^{* *}$ M. Vaccarini* \\ * Università Politecnica delle Marche, Ancona, Italy, \\ sauro.longhi@univpm.it,m.vaccarini@diiga.univpm.it \\ ** API Oil Industry, Italy, r.trillini@apioil.com
}

\begin{abstract}
The performance of a networked decentralized model predictive control is analyzed and tested on an industrial process characterized by strong interactions. The proposed control architecture is based on a set of independent agents which implement independent model predictive control strategies and exchange a reduced set of information by a local network. The experimental results are satisfactory and comparable with those obtained by the classical centralized model predictive control strategy. Copyright (c) 2005 IFAC
\end{abstract}

Keywords: model based control, predictive control, decentralized control, networks, industrial production systems.

\section{INTRODUCTION}

Production processes are generally composed by different plants, which are interconnected and characterized by significant interactions. In general, a simple control solution for the regulation of these plants based on independent controllers does not guarantee the overall process performance and stability. Therefore for these processes two different solutions can be developed: a centralized control solution, where all the interactions are considered, or a decentralized control solution where each independent control agent is able to acquire estimations or measurements of the interactions.

In this paper the use of a model predictive control strategy is proposed for the control agents operating in a decentralized control architecture. In general, the optimum policy for each agent does not guarantee the global optimum. Global objectives, such as closed-loop stability or some performance requirements for the global process require coordination among the control agents.
The required coordination can be introduced by a hierarchical decentralized control scheme, where a supervisor computes the global optimum and coordinates all the control agents. In this solution the reliability is low, the quantity of information to be exchanged is high and the supervisor velocity to react to parameters changes is low, because it works at a low sampling frequency (Šiljak, 1996). A decentralized filtering and control architecture managed by a global coordinator has been proposed in (Katebi and Johnson, 1997) for guaranteing steady state optimality of the control actions. A different solution based on the team theory and asynchronous teams has been recently proposed in (Camponogara, 2000). In (Jia, 2003) the global process is decomposed into subsystems based on the structural properties and physical constraints and each agent controls one of them making use of local model, objectives and constraints. In the developed solutions satisfactory performances are guaranteed in the case of weak interactions among subsystems. Recently, networked distributed control systems for chemical plants have been also developed with signif- 


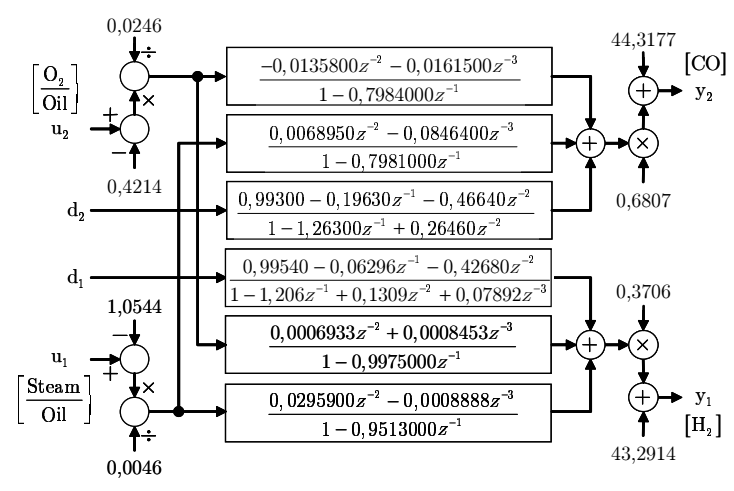

Fig. 1. Identified gasifier model.

icant results and potential new implementation issues (El-Farra et al., 2004).

In this paper a contribution to decentralized implementation of model predictive control strategy is proposed. The innovative solution is based on independent agents and on a local network used for exchanging a reduced set of information. This Networked Decentralized Model Predictive Control architecture (in the following referred as NDMPC), guarantees satisfactory performance also in the case of strong interactions among subsystems. The performance of the proposed solution is tested on a process characterized by strong interactions and here assumed as a case study.

\section{CASE STUDY}

In this paper a gasification process is considered for testing the proposed networked decentralized model predictive control. In an Oil Industry the gasification process is the main part of a Syngas Manufacturing Process Plant (SMPP). It consists in the production of syngas from the reaction between Oxygen, Steam and the Charge Oil (Oil), that is a visbreaking residual composed by heavy hydrocarbons. The syngas, which is rich of $\mathrm{H}_{2}, \mathrm{CO}$ and other hydrocarbons, is used in a Combined Cycle Power Plant (CCPP) for steam and power generation. The control objective for this process is the maximization of the Cold Gas Efficiency (CGE) and the minimization of the Specific Oxygen Consumption (SOC). CGE and SOC both depend on the rate of $\mathrm{H}_{2}$ and $\mathrm{CO}$ in the syngas.

Based on these definitions, $\mathrm{O}_{2} / \mathrm{Oil}$ and Steam/Oil ratios are considered as manipulated variables which have to be imposed in order to obtain the desired values for the controlled variables $\mathrm{H}_{2}$ and CO. To optimize CGE and SOC, a hierarchical control structure is considered. In this structure the reference values of $\mathrm{H}_{2}$ and $\mathrm{CO}$ are imposed by an higher controller and the control actions $\mathrm{O}_{2} /$ Oil and Steam/Oil ratios are computed by a lower controller which implements the proposed networked distributed architecture.
For a given composition range of the Oil, a linear dynamic model of this process has been developed by means of an identification procedure based on historical data of the gasifier, (Barboni, 2002). The identified input-output model is shown in Figure 1 . In addition to the manipulated variables $\mathrm{O}_{2} /$ Oil and Steam/Oil, two disturbance inputs $d_{1}$ and $d_{2}$ are introduced for modelling the disturbances and other dynamics. Furthermore, a sampling period $T_{c}=300 \mathrm{~s}$ is imposed and the following input and output ranges are considered: $0,390 \leq$ Steam/Oil $\leq 0,449,1,0453 \leq$ $\mathrm{O}_{2} / \mathrm{Oil} \leq 1,0636,42,50 \% \mathrm{Vol} \leq \mathrm{H}_{2} \leq 44,30 \% \mathrm{Vol}$, $44,07 \% \mathrm{Vol} \leq \mathrm{CO} \leq 46,00 \% \mathrm{Vol}$.

\section{CENTRALIZED MPC}

Model predictive control (MPC) is a control technique based on the receding horizon principle that is also known as receding horizon control (RHC) (Soeterboek, 1992). At each sampling time, using a predictive model of the system dynamics, the response of the process to changes in manipulated variables over a fixed horizon $p$ (called the prediction horizon) is predicted. Based on a proper objective function, a finite-horizon optimal control problem is solved to obtain current and future moves of the manipulated variables. Only the first computed move is actually applied to the real system whereas all other control actions in the optimal control sequence are discarded. The same procedure is repeated at the next control step based on the new measurement (García et al., 1989; Qin and Badgwell, 2000; Rawlings, 2000).

Denoting by $\mathbf{y} \in \mathbb{R}^{n_{y}}$ the process output vector, $\mathbf{r} \in \mathbb{R}^{n_{y}}$ the reference vector, $\mathbf{u} \in \mathbb{R}^{n_{u}}$ the control input vector, $\mathbf{z} \in \mathbb{R}^{n_{z}}$ the measurable disturbance vector, $\hat{\mathbf{x}}(k) \in \mathbb{R}^{n}$ the initial state estimation and $\boldsymbol{\Delta} \mathbf{u}(k \mid k) \triangleq \mathbf{u}(k \mid k)-\mathbf{u}(k-1 \mid k)$ the control input move, the standard MPC optimization problem at control step $k$ is

$$
\min _{\Delta \mathbf{u}(k \mid k), \ldots, \boldsymbol{\Delta} \mathbf{u}(k+m-1 \mid k)}
$$

where

$$
\begin{aligned}
J=\sum_{l=1}^{p} \| \hat{\mathbf{y}}( & k+l \mid k)-\mathbf{r}(k+l) \|_{\boldsymbol{\Gamma}_{y}^{l}}+ \\
& +\sum_{l=1}^{m}\|\boldsymbol{\Delta} \mathbf{u}(k+l-1 \mid k)\|_{\boldsymbol{\Gamma}_{u}^{l}},
\end{aligned}
$$

subject to model constraints

$$
\begin{gathered}
\hat{\mathbf{x}}(k+l \mid k)=\mathbf{f}(\hat{\mathbf{x}}(k+l-1 \mid k), \\
\mathbf{u}(k+l-1 \mid k), \mathbf{z}(k+l-1)) \\
\hat{\mathbf{y}}(k+l \mid k)=\mathbf{g}(\hat{\mathbf{x}}(k+l \mid k), \\
\mathbf{u}(k+l \mid k), \mathbf{z}(k+l)) \\
l=1, \ldots, p
\end{gathered}
$$


and operating constraints

$$
\begin{array}{ll}
\mathbf{y}_{l}^{\min } \leq \hat{\mathbf{y}}(k+l \mid k) \leq \mathbf{y}_{l}^{\max } & l=1, \ldots, p \\
|\boldsymbol{\Delta} \mathbf{u}(k+l \mid k)| \leq \boldsymbol{\Delta} \mathbf{u}_{l}^{\max } & l=1, \ldots, m \\
\mathbf{u}_{l}^{\min } \leq \mathbf{u}(k+l \mid k) \leq \mathbf{u}_{l}^{\max } & l=1, \ldots, m
\end{array}
$$

where $m \in \mathbb{N}(m \leq p)$ is called the control horizon.

Stability, feasibility and robustness problems have been analyzed and different contributions are proposed in literature (Cheng and Krogh, 2001; Bemporad and Morari, 1999; Mayne et al., 2000).

\section{NETWORKED DECENTRALIZED MPC}

In this paper a NDMPC is analyzed where the control actions are computed by a set of subcontrollers which are independent agents able to dynamically exchange a restrict set of information. This control architecture is applied to a process where in general there are strong interactions among the $M$ subsystems $S_{i}$ characterized by the input $u_{i}$ and the output $y_{i}, i=1, \ldots, M$. Each agent $A_{i}$ implements a MPC algorithm for the subsystem $S_{i}$ where the optimization problem is solved making use of local information acquired on $S_{i}$ and the estimate of the interactions among $S_{i}$ with the other subsystems $S_{j}, j=1, \ldots, M$, $j \neq i$. In particular, the future state trajectories and the future control actions over the prediction horizon have to be exchanged among subsystems. The agents $A_{j}$ which exchange information with $A_{i}$ are called neighboring agents of $A_{i}$. A local area network is used for data exchanging.

The agent $A_{i}$ is composed of two parts: an optimizer and an estimator. Based on the exchanged information the estimator produces an interaction estimate which is used together with local measurements by the optimizer to solve the MPC optimization problem and to estimate the state predictions. The first control action of the optimal sequence is applied to the subsystem $S_{i}$ and the optimal control sequence and the state predictions over the control horizon are transmitted to the neighboring agents by the local area network. This strategy leads to a completely distributed coordinated control scheme (Jia and Krogh, 2001; Camponogara et al., 2002).

It is assumed that the agents are synchronous and that the communication channel introduces a delay of one sampling period. Therefore the exchanged information are delayed and referred to the previous sampling instant and each agent can exchange information with the other agents only one time for each sampling interval.

Assuming, for the sake of simplicity but without loss of generality, that the prediction and control horizons are the same for each agent, in NDMPC the optimization problem of each agent $A_{i}$ at control step $k$ can be stated by relations similar to (1), (2) and (3) where disturbance vector $\mathbf{z}$ includes, in this case, the interactions with the other subsystems $S_{j}, j \neq i$. The problem here considered is stated in the following subsection.

\subsection{Control problem formulation}

Consider the linear discrete time process described by:

$$
\begin{aligned}
\mathbf{x}(k+1) & =\mathbf{A} \mathbf{x}(k)+\mathbf{B u}(k)+\mathbf{E w}(k) \\
\mathbf{y}(k) & =\mathbf{C x}(k)+\mathbf{F} \mathbf{v}(k)
\end{aligned}
$$

where $\mathbf{x}(k) \in \mathbb{R}^{n}$ is the state vector and $\mathbf{w}(k) \in$ $\mathbb{R}_{w}^{n}$ and $\mathbf{v}(k) \in \mathbb{R}^{n_{v}}$ are white gaussian noises with zero mean value and covariance matrixes $\mathbf{Q}$ and $\mathbf{R}$ respectively. If $\mathbf{E}$ and $\mathbf{F}$ are diagonal block matrices, the overall system (5) can always be decomposed in $M$ subsystems $S_{i}, i=1, \ldots, M$ of the form

$$
\begin{array}{r}
\mathbf{x}_{i}(k+1)=\mathbf{A}_{i} \mathbf{x}_{i}(k)+\mathbf{B}_{i} \mathbf{u}_{i}(k)+\mathbf{E}_{i} \mathbf{w}_{i}(k)+ \\
+\mathbf{G}_{i} \mathbf{z}_{i}(k) \\
\mathbf{y}_{i}(k)=\mathbf{C}_{i} \mathbf{x}_{i}(k)+\mathbf{F}_{i} \mathbf{v}_{i}(k)+\mathbf{H}_{i} \mathbf{z}_{i}(k)
\end{array}
$$

where $\mathbf{z}_{i}(k) \in \mathbb{R}^{n_{z_{i}}}$ is the interaction vector, $\mathbf{x}_{i}(k) \in \mathbb{R}^{n_{i}}, \mathbf{u}_{i}(k) \in \mathbb{R}^{n_{u_{i}}}, \mathbf{y}_{i}(k) \in \mathbb{R}^{n_{y_{i}}}$ are the state vector, the manipulated variables vector and the output vector of $S_{i}$ respectively. Since matrices $\mathbf{E}$ and $\mathbf{F}$ are supposed diagonal block, the stochastic disturbances $\mathbf{w}_{i}(k) \in \mathbb{R}^{n_{w_{i}}}$ and $\mathbf{v}_{i}(k) \in \mathbb{R}^{n_{v_{i}}}$ affects only the subsystem $S_{i}$. This hypothesis is not too restrictive in a distributed framework because is highly improbable that the same disturbance affects two far areas. The interaction vector $\mathbf{z}_{i}(k)$ contains both the state and the input vector of the other subsystems $S_{j}, j \neq i$ :

$$
\begin{gathered}
\mathbf{z}_{i}(k) \triangleq\left[\mathbf{u}_{1}^{\prime}(k) \cdots \mathbf{u}_{i-1}^{\prime}(k) \mathbf{u}_{i+1}^{\prime}(k) \cdots \mathbf{u}_{M}^{\prime}(k)\right. \\
\left.\mathbf{x}_{1}^{\prime}(k) \cdots \mathbf{x}_{i-1}^{\prime}(k) \mathbf{x}_{i+1}^{\prime}(k) \cdots \mathbf{x}_{M}^{\prime}(k)\right]^{\prime}
\end{gathered}
$$

Note that the non zero blocks of the row block matrices $\mathbf{G}_{i}$ and $\mathbf{H}_{i}$ characterize the interactions with the other subsystems $S_{j}$ and then the number of neighboring agents of $A_{i}$. At the sampling instant $k$, neither $\mathbf{u}_{j}(k)$ nor $\mathbf{x}_{j}(k)$ for $j \neq i$ are available by the agent $A_{i}$ but only their predictions previously sent by the agent $A_{j}$ are known. The state $l$-step ahead prediction to be used in the MPC strategy is:

$$
\begin{gathered}
\hat{\mathbf{x}}_{i}(k+l \mid k)=\mathbf{A}_{i}^{l} \hat{\mathbf{x}}_{i}(k \mid k)+ \\
+\sum_{s=1}^{l} \mathbf{A}_{i}^{s-1}\left[\mathbf{B}_{i} \mathbf{u}_{i}(k+l-s \mid k)+\right. \\
\left.\quad+\mathbf{G}_{i} \hat{\mathbf{z}}_{i}(k+l-s \mid k-1)\right] .
\end{gathered}
$$

where $\hat{\mathbf{x}}_{i}(k \mid k)$ is the estimate of $\mathbf{x}_{i}(k)$ at the time $k$ and $\hat{\mathbf{z}}_{i}(k+l-s \mid k-1)$ is the prediction of $\mathbf{z}_{i}(k+l-s)$ computed at the time $k-1$. 
By equation (5b), the corresponding output $l$-step ahead prediction has the following form:

$$
\begin{aligned}
\hat{\mathbf{y}}_{i}(k+l \mid k)=\mathbf{C}_{i} \hat{\mathbf{x}}_{i}( & k+l \mid k)+ \\
& +\mathbf{H}_{i} \hat{\mathbf{z}}_{i}(k+l \mid k-1) .
\end{aligned}
$$

In the receding horizon strategy, only the first element of the optimal control sequence $\mathbf{u}_{i}(k \mid k)$ will be applied to the process whereas the other ones will be recomputed at the next control instants. Thus agent $A_{i}$ sends to the neighboring agents the computed values $\mathbf{u}_{i}(k+l \mid k)$ and $\hat{\mathbf{x}}_{i}(k+l \mid k)$ for $l=1, \ldots, p$ that during the transient behavior have a low reliability. In general this fact reduces the performances of the overall system. Therefore the MPC strategy of agent $A_{i}$ has to be modified in order to guarantee that at least the next control action $\mathbf{u}_{i}(k+1 \mid k)$ will be actually applied to the process. This solution requires an integration on the coordination strategy that is described in the following subsection.

\subsection{Completely distributed coordination strategy}

The distributed coordination can be achieved assuming that, at time $k$, the agent $A_{i}$ applies the previously computed control action $\mathbf{u}_{i}(k \mid k-1)$ which has been sent to the neighboring agents and computes the future control actions $\mathbf{u}_{i}(k+1 \mid k), \ldots, \mathbf{u}_{i}(k+m \mid k)$. This solution improves the control performances because each agent can compute its predictions and moves based on real input data. The drawback is that this technique increases the reaction time of one sampling period.

The agent $A_{i}$ considers a functional to be optimized of the form

$$
\begin{aligned}
J_{i}=\sum_{l=1}^{p}\left\|\hat{\mathbf{y}}_{i}(k+l \mid k)-\mathbf{r}_{i}(k+l)\right\|_{\boldsymbol{\Gamma}_{y_{i}}^{l}}+ \\
+\sum_{l=1}^{m}\left\|\boldsymbol{\Delta} \mathbf{u}_{i}(k+l \mid k)\right\|_{\boldsymbol{\Gamma}_{u_{i}}^{l}},
\end{aligned}
$$

where $\boldsymbol{\Delta} \mathbf{u}_{i}(k \mid k) \triangleq \mathbf{u}_{i}(k \mid k)-\mathbf{u}_{i}(k-1 \mid k)$. Defined the vector of output prediction by

$$
\hat{\mathbf{Y}}_{i}(k+1 \mid k) \triangleq\left[\hat{\mathbf{y}}_{i}^{\prime}(k+1 \mid k) \cdots \hat{\mathbf{y}}_{i}^{\prime}(k+p \mid k)\right]^{\prime},
$$

it can be expressed as

$$
\hat{\mathbf{Y}}_{i}(k+1 \mid k)=\mathbf{L}_{i}+\mathbf{M}_{i} \Delta \mathbf{U}_{i}(k)
$$

where

$$
\boldsymbol{\Delta} \mathbf{U}_{i}(k) \triangleq\left[\boldsymbol{\Delta} \mathbf{u}_{i}^{\prime}(k+1 \mid k) \cdots \boldsymbol{\Delta} \mathbf{u}_{i}^{\prime}(k+m \mid k)\right]^{\prime}
$$

and matrix $\mathbf{M}_{i}$ depends on the model matrices $\mathbf{A}_{i}, \mathbf{B}_{i}$ and $\mathbf{C}_{i}$, while matrix $\mathbf{L}_{i}$ depends on $\hat{\mathbf{x}}_{i}(k \mid k-1), \mathbf{y}_{i}(k), \mathbf{u}_{i}(k \mid k-1)$ and $\mathbf{z}_{i}(k \mid k-1)$, $\ldots, \mathbf{z}_{i}(k+p \mid k-1)$.

Using the relation (10), the objective function (9) of $A_{i}$ takes the following form:

$$
\begin{aligned}
J_{i}=\Delta \mathbf{U}_{i}^{\prime}(k) & \mathcal{H}_{i} \Delta \mathbf{U}_{i}(k)+ \\
& -\mathcal{G}_{i}^{\prime}(k+1 \mid k) \Delta \mathbf{U}_{i}(k),
\end{aligned}
$$

where

$$
\begin{aligned}
\mathcal{G}_{i}(k+1 \mid k) & \triangleq 2 \mathbf{M}_{i}^{\prime} \boldsymbol{\Gamma}_{Y_{i}}\left[\mathbf{R}_{i}(k+1 \mid k)-\mathbf{L}_{i}\right] \\
\mathcal{H}_{i} & \triangleq \mathbf{M}_{i}^{\prime} \boldsymbol{\Gamma}_{Y_{i}} \mathbf{M}_{i}+\boldsymbol{\Gamma}_{U_{i}} .
\end{aligned}
$$

with $\boldsymbol{\Gamma}_{Y_{i}} \triangleq \operatorname{diag}\left\{\boldsymbol{\Gamma}_{y_{i}}^{1}, \ldots, \boldsymbol{\Gamma}_{y_{i}}^{p}\right\}$ and $\boldsymbol{\Gamma}_{U_{i}} \triangleq$ $\operatorname{diag}\left\{\boldsymbol{\Gamma}_{u_{i}}^{1}, \ldots, \boldsymbol{\Gamma}_{u_{i}}^{m}\right\}$ assigned and $\mathbf{R}_{i}(k+1 \mid k) \triangleq$ $\left[\mathbf{r}_{i}^{\prime}(k+1) \cdots \mathbf{r}_{i}^{\prime}(k+p)\right]^{\prime}$. For each agent $A_{i}$, input and output constraints can be expressed in terms of $\boldsymbol{\Delta} \mathbf{U}_{i}(k)$ and, for each $i=1, \ldots, M$, the optimization problem associated with the considered NDMPC strategy can be stated as follows:

$$
\begin{aligned}
\min _{\Delta \mathbf{U}_{i}(k)} \boldsymbol{\Delta} \mathbf{U}_{i}^{\prime}(k) & \mathcal{H}_{i} \boldsymbol{\Delta} \mathbf{U}_{i}(k)+ \\
& -\mathcal{G}_{i}^{\prime}(k+1 \mid k) \Delta \mathbf{U}_{i}(k) \\
\text { s.t. } & \boldsymbol{\Phi}_{i} \boldsymbol{\Delta} \boldsymbol{U}_{i}(k) \geq \boldsymbol{\varphi}_{i} .
\end{aligned}
$$

where $\boldsymbol{\Phi}_{i}$ is a suitable matrix composed by $\mathbf{M}_{i}$ and some identity blocks while $\boldsymbol{\varphi}_{i}$ is a vector containing the limits $\mathbf{y}_{i, l}^{\min }, \mathbf{y}_{i, l}^{\max }, \mathbf{u}_{i, l}^{\min }, \mathbf{u}_{i, l}^{\max }$ and $\boldsymbol{\Delta} \mathbf{u}_{i, l}^{\max }$ of the local constraints. Equations (13a) and (13b) define a quadratic program which has to be solved on-line at every sampling instant.

In order to test the performances of the proposed NDMPC for highly coupled subsystems interacting also through input variables, this control strategy is applied to the gasificator introduced in Section 2 as a case study.

\section{RESULTS}

Performances of the distributed control strategy have been tested by numerical simulations and compared with the best solution obtained by the classical centralized MPC strategy where all information are available for the computation of the optimal solution.

The estimated linear MIMO input-output model of the considered plant, that is schematically shown in Figure 1, can be stated in the state space form (2) with $\mathbf{v}(k)=\mathbf{w}(k)=\left[d_{1}(k) d_{2}(k)\right]^{\prime}$, $\mathbf{u}(k)=\left[u_{1}(k) u_{2}(k)\right]^{\prime}, \mathbf{y}(k)=\left[y_{1}(k) y_{2}(k)\right]^{\prime}$, state space dimension $n=17$ and

$$
\begin{aligned}
& \mathbf{A}=\operatorname{diag}\left\{\boldsymbol{\alpha}_{1}, \boldsymbol{\alpha}_{2}, \boldsymbol{\alpha}_{3}, \boldsymbol{\alpha}_{4}, \boldsymbol{\alpha}_{5}, \boldsymbol{\alpha}_{6}\right\} \\
& \mathbf{B}=\left[\begin{array}{ll}
\boldsymbol{\beta}_{11} & \boldsymbol{\beta}_{12} \\
\boldsymbol{\beta}_{21} & \boldsymbol{\beta}_{22}
\end{array}\right] \quad \mathbf{C}=\operatorname{diag}\left\{\boldsymbol{\gamma}_{1}, \boldsymbol{\gamma}_{2}\right\} \\
& \mathbf{E}=\operatorname{diag}\left\{\boldsymbol{\epsilon}_{1}, \boldsymbol{\epsilon}_{2}\right\} \quad \mathbf{F}=\operatorname{diag}\left\{\boldsymbol{\phi}_{1}, \boldsymbol{\phi}_{2}\right\}
\end{aligned}
$$

where

$$
\begin{aligned}
\boldsymbol{\alpha}_{1} & =\left[\begin{array}{ccc}
0.9513 & 0 & 0 \\
1 & 0 & 0 \\
0 & 1 & 0
\end{array}\right] \quad \boldsymbol{\alpha}_{2}=\left[\begin{array}{ccc}
0.9975 & 0 & 0 \\
1 & 0 & 0 \\
0 & 1 & 0
\end{array}\right] \\
\boldsymbol{\alpha}_{3} & =\left[\begin{array}{ccc}
1.2060 & -0.1309 & -0.0789 \\
1 & 0 & 0 \\
0 & 1 & 0
\end{array}\right] \\
\boldsymbol{\alpha}_{4} & =\left[\begin{array}{ccc}
0.7981 & 0 & 0 \\
1 & 0 & 0 \\
0 & 1 & 0
\end{array}\right] \quad \boldsymbol{\alpha}_{5}=\left[\begin{array}{ccc}
0.7984 & 0 & 0 \\
1 & 0 & 0 \\
0 & 1 & 0
\end{array}\right] \\
\boldsymbol{\alpha}_{6} & =\left[\begin{array}{ccc}
1.2630 & -0.2646 \\
1 & 0
\end{array}\right]
\end{aligned}
$$



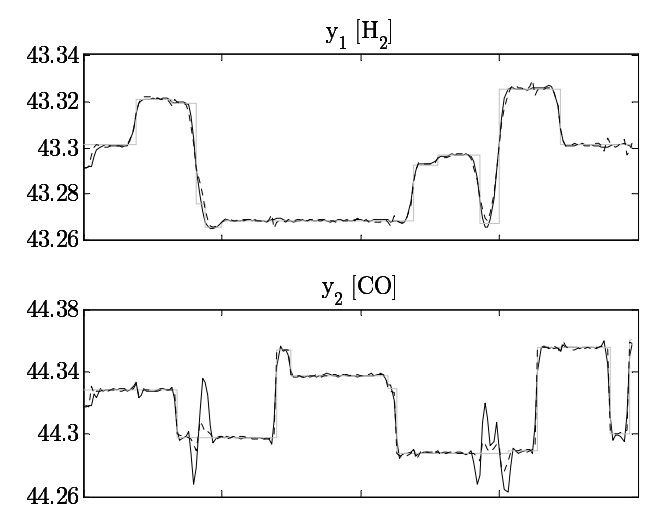

44.2
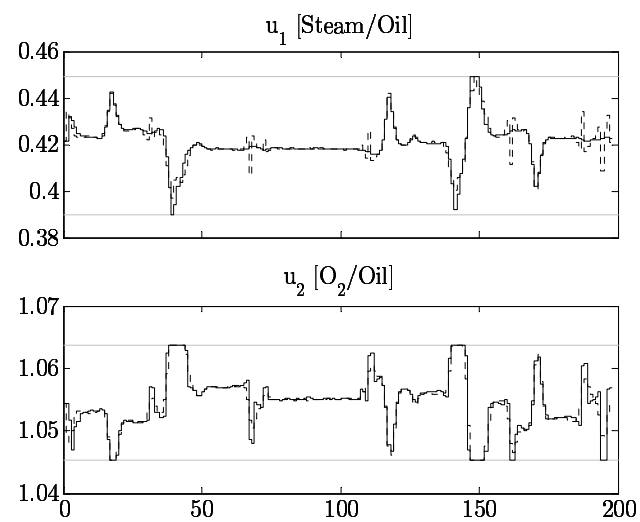

Fig. 2. Constrained NDMPC with agents implementing the proposed completely distributed coordination strategy (solid line) and classical centralized MPC (dashed line).
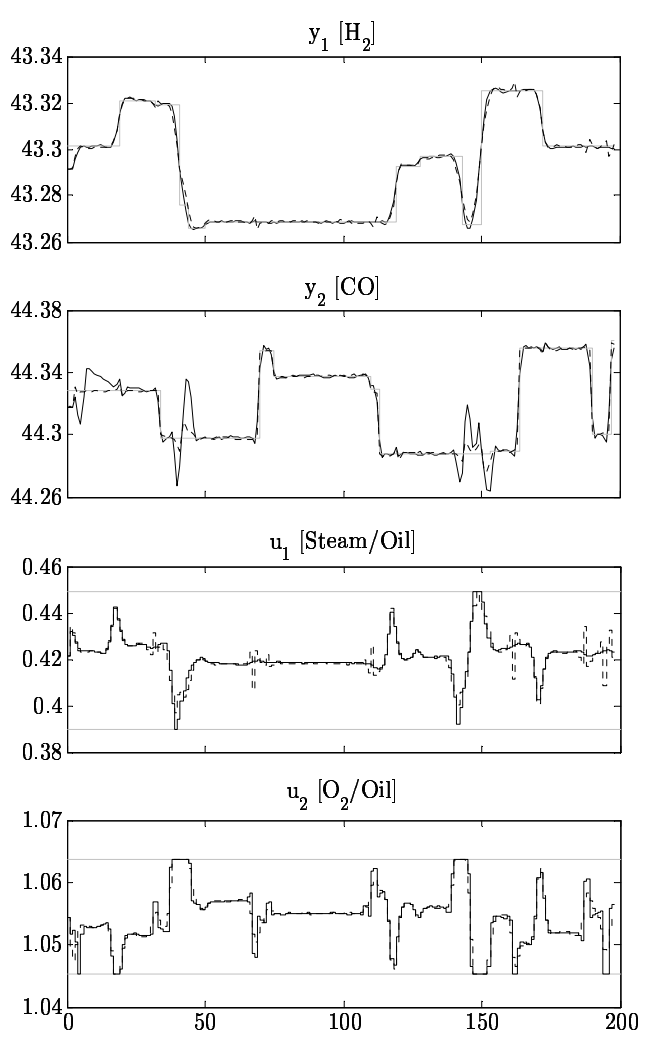

Fig. 3. Constrained NDMPC with agents implementing the simple coordination strategy (solid line) and classical centralized MPC (dashed line).
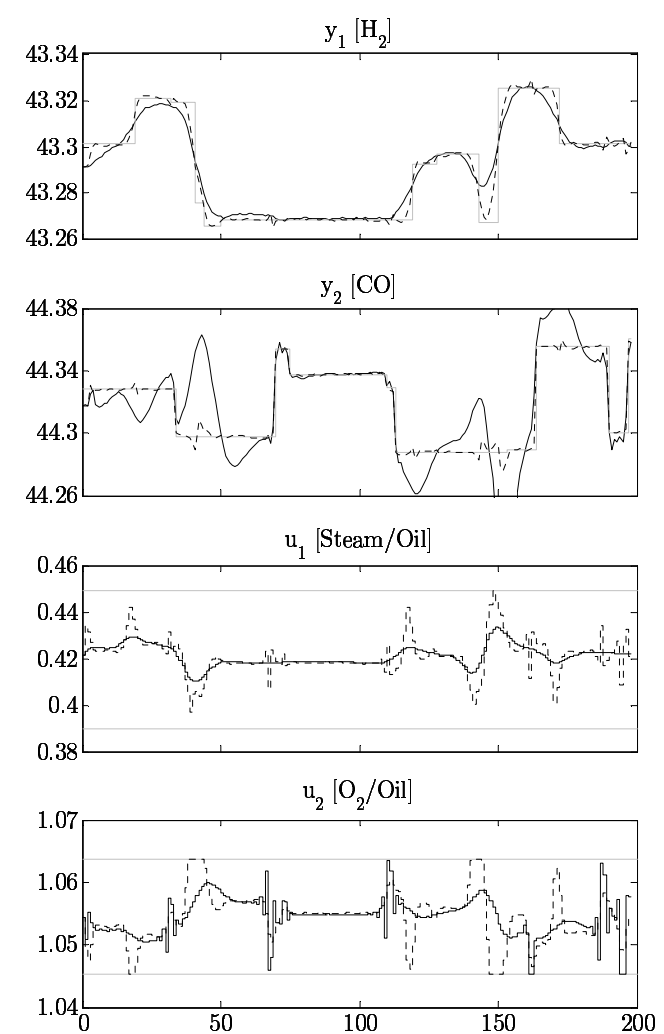

Fig. 4. Constrained DMPC with independent agents implementing a decentralized MPC without networked data exchange (solid line) and classical centralized MPC (dashed line).

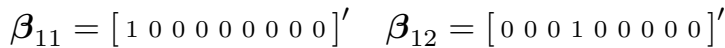

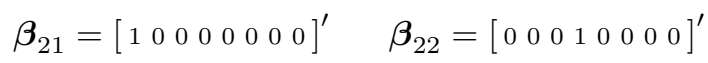

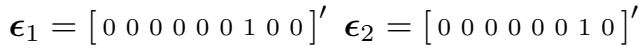

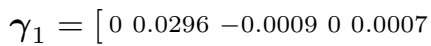

$$
\begin{aligned}
& 0.00081 .1375-0.5571-0.0786] \\
& \gamma_{2}=\left[\begin{array}{lllll}
0 & 0.0069 & -0.0846 & 0 & -0.0136
\end{array}\right. \\
& -0.01621 .0579-0.7291] \\
& \phi_{1}=0.9954 \quad \phi_{2}=0.9930 \text {. }
\end{aligned}
$$

The plant input-output relationship analysis provided that input $u_{1}$ and $u_{2}$ are strongly interactive with output $y_{1}$ and $y_{2}$ respectively. Therefore the plant has been decomposed in two subsystems $S_{i}$ with input $u_{i}$ and output $y_{i}, i=1,2$, described by the state space form (5) where

$$
\begin{aligned}
& \mathbf{A}_{1}=\operatorname{diag}\left\{\boldsymbol{\alpha}_{1}, \boldsymbol{\alpha}_{2}, \boldsymbol{\alpha}_{3}\right\} \quad \mathbf{B}_{1}=\boldsymbol{\beta}_{11} \quad \mathbf{C}_{1}=\boldsymbol{\gamma}_{1} \\
& \mathbf{E}_{1}=\boldsymbol{\epsilon}_{1} \quad \mathbf{F}_{1}=\phi_{1} \quad \mathbf{G}_{1}=\boldsymbol{\beta}_{12} \quad \mathbf{H}_{1}=\mathbf{0}, \\
& \mathbf{A}_{2}=\operatorname{diag}\left\{\boldsymbol{\alpha}_{4}, \boldsymbol{\alpha}_{5}, \boldsymbol{\alpha}_{6}\right\} \quad \mathbf{B}_{2}=\boldsymbol{\beta}_{22} \quad \mathbf{C}_{2}=\boldsymbol{\gamma}_{2} \\
& \mathbf{E}_{2}=\boldsymbol{\epsilon}_{2} \quad \mathbf{F}_{2}=\phi_{2} \quad \mathbf{G}_{2}=\boldsymbol{\beta}_{21} \quad \mathbf{H}_{2}=\mathbf{0} .
\end{aligned}
$$

The interaction between subsystems $S_{1}$ and $S_{2}$ is given by the manipulated variables and this allows to test the effectiveness of the proposed coordination strategy. The range of validity of the considered plant model requires to impose the set of constraints stated in Section 2.

In all the plotted results, the outputs $\left[\mathrm{H}_{2}\right]$ and $[\mathrm{CO}]$ are measured in percentage of vol- 
ume $(\% \mathrm{Vol})$, the control inputs [Steam/Oil] and $\left[\mathrm{O}_{2} / \mathrm{Oil}\right]$ are adimensional quantities and the labels in the time axis represent the sample instants. Moreover two gaussian noises have been applied to the inputs $d_{1}$ and $d_{2}$ and all the weighting matrices of the controllers have been tuned in order to obtain the best possible performances.

Behaviors of the NDMPC strategy proposed in Subsection 4.2 are shown in Figure 2 for a reference trajectory composed by a random sequence of steps (gray line). The behaviors of the classical centralized MPC strategy are also represented by the dashed lines. With the introduced networked decentralized control strategy based on two independent agents, the control performances are similar to those obtained by the classical centralized MPC strategy that represents the best solution.

Defining the output errors $e_{i}(k) \triangleq y_{i}(k)-r_{i}(k)$, $i=1,2$, the mean square output errors $\bar{e}_{i}^{a}$, $i=1,2$ obtained with the completely distributed coordination strategy are $\bar{e}_{1}^{a}=1.4 \cdot 10^{-5}$ and $\bar{e}_{2}^{a}=6.3 \cdot 10^{-5}$, respectively, that are similar to the mean square output errors $\bar{e}_{i}^{*}$ obtained with the classical centralized MPC: $\bar{e}_{1}^{*}=1.7 \cdot 10^{-5}$, $\bar{e}_{2}^{*}=0.7 \cdot 10^{-5}$. Figure 3 shows the behavior of a distributed coordination without the completely distributed coordination strategy between agents $A_{1}$ and $A_{2}$ stated in Subsection 4.2. This solution introduces a slight loss of performances during the transients behavior but it is more reactive. In fact the mean square output errors $\bar{e}_{i}^{b}$ obtained with this control strategy are similar to that of the proposed coordination strategy $\left(\bar{e}_{1}^{b}=1.4 \cdot 10^{-5}\right.$, $\bar{e}_{2}^{b}=6.4 \cdot 10^{-5}$ ) but the corresponding overshots are relatively bigger. The performances of a couple of non interacting agents which implement a DMPC without networked data exchange, are shown in Figure 4. In this case the absence of communication causes a significant degradation of the closed loop response and the control errors $\bar{e}_{i}^{c}$ are $\bar{e}_{1}^{c}=4.3 \cdot 10^{-5}, \bar{e}_{2}^{c}=41.0 \cdot 10^{-5}$.

Similar results are obtained in a wide set of experimental tests which are summarized in Table 1 for five reference sequences with increasing width.

Table 1. Percent mean square output errors referred to the mean square output errors of the classical centralized MPC.

\begin{tabular}{rrrrrr}
\multicolumn{7}{c}{ Subsystem $S_{1}$} \\
\hline & I & II & III & IV & V \\
\hline $\bar{e}_{1}^{a} \%$ & 0 & -3 & -9 & -14 & -22 \\
$\bar{e}_{1}^{b} \%$ & -4 & -7 & -14 & -20 & -23 \\
$\bar{e}_{1}^{c} \%$ & 267 & 324 & 211 & 139 & 134 \\
\hline
\end{tabular}

\begin{tabular}{lrrrrr}
\multicolumn{7}{c}{ Subsystem $S_{2}$} \\
\hline & $\mathrm{I}$ & $\mathrm{II}$ & $\mathrm{III}$ & $\mathrm{IV}$ & $\mathrm{V}$ \\
\hline $\bar{e}_{2}^{a} \%$ & 51 & 109 & 651 & 630 & 678 \\
$\bar{e}_{2}^{b} \%$ & 259 & 154 & 939 & 847 & 724 \\
$\bar{e}_{2}^{c} \%$ & 8512 & 7569 & 8243 & 6259 & 4567 \\
\hline
\end{tabular}

\section{CONCLUSIONS}

The preliminary results obtained by the proposed NDMPC are satisfactory if compared with those obtained by the classical centralized MPC. Good performances are obtained by data exchanging among the control agents which allows to produce a local estimate of the mutual interactions. The analysis of the closed loop stability is under investigations by the extension to the considered case of analysis results stated in (Jia, 2003). Moreover, also robustness results for the proposed control solution are needed in order to handle model uncertainties and parameter variations. In particular, under bounded uncertainties, a min-max optimization problem can be considered and a robust control action can be found by the minimization of the objective function for that value of uncertainties which maximizes the objective function.

\section{REFERENCES}

Barboni, L. (2002). Modello ibrido di un impianto di gassificazione. Degree thesis, Università Politecnica delle Marche, (Italian language).

Bemporad, A. and M. Morari (1999). Robust model predictive control: A survey. In: Robustness in Identification and Control, Lecture Notes in Control and Information Sciences (A. Garulli, A. Tesi and A. Vicino, Eds.). Vol. 245. pp. 207-226. Springer. London.

Camponogara, E. (2000). Controlling networks with collaborative agents. Ph.D. thesis, Carnegie Mellon Univ.

Camponogara, E., D. Jia, B.H. Krogh and S. Talukdar (2002). Distributed model predictive control. IEEE Control Syst. Mag. 22, 44-52.

Cheng, X. and B.H. Krogh (2001). Stability-constrained model predictive control. IEEE Trans. on Automatic Control 46, 1816-1820.

El-Farra, N.H., A. Gani and P.D. Christofides (2004). Fault-tolerant control of multi-unit process systems using communication networks. Proc. of 7 th IFAC Symposium on Dynamics and Control of Proc.Syst.

García, C.E., D.M. Prett and M. Morari (1989). Model predictive control: theory and practice - a survey. Automatica 25, 335-348.

Jia, D. (2003). Distributed coordination in multiagent control systems through model predictive control. Ph.D. thesis, Carnegie Mellon Univ.

Jia, D. and B.H. Krogh (2001). Distributed model predictive control. Proc. of the American Control Conference 4, 2767-2772.

Katebi, M.R. and M.A. Johnson (1997). Predictive control design for large-scale systems. Automatica 33, 421425 .

Mayne, D.Q., J.B. Rawlings, C.V. Rao and P.O.M. Scokaert (2000). Constrained model predictive control: Stability and optimality. Automatica 36, 789814 .

Qin, S.J. and T.A. Badgwell (2000). An overview of nonlinear model predictive control applications. In: Nonlinear Predictive Control (F. Allgöwer and A. Zheng, Eds.). pp. 369-392. Birkhäuser.

Rawlings, J.B. (2000). Tutorial overview of model predictive control. IEEE Control Syst. Mag. 20, 38-52.

Soeterboek, R. (1992). Predictive Control. A Unified Approach. Prentice Hall. New York.

Šiljak, D.D. (1996). Decentralized control and computations: status and prospects. Annual Reviews in Control 20, 131-141. 